Ann. Biol. anim. Bioch. Biophys., I962, 2 (2), II9-127

\title{
ÉVOLUTION D'UNE FRÉQUENCE GÉNIQUE DANS LA RACE OVINE «BLEU DU MAINE»
}

\author{
J. J. IALVERGNE \\ Station de Génétique animale, \\ Centre national de Recherches zootechniques, Jour-en-Josas (Seine t Oise).
}

\section{SOMMAIRE}

Le gène récessif $b$ qui existe dans la race ovine "Bleu du Naine " est jugé indésirable, car il détermine la couleur noire de la laine. Malgré l'élimination systématique des animaux homozygotes récessifs, cet allèle subsiste dans les troupeaux.

Dans le troupeau Chollet-Molines, on montre que la persistance du gène $b$ peut s'expliquer par un modèle de génétique des populations dans lequel tous les mâles seraient hétérozygotes, aucune sélection ne s'effectuant chez les femelles pour différencier les sujets $+/ b$ des $+/+$. Un test appliqué à 3 générations successives d'individus n'infirme pas notre hypothèse ;'la fréquence génique trouvée est alors voisine de la fréquence d'équilibre 0,30 , ce qui conduit à $5 \mathrm{p}$. 1oo de naissances noires.

La sélection préférentielle effectuée chez les mâles pour le génotype + /b s'expliquerait par le fait qu'à l'état hétérozygote $b$ aviverait la coloration bleue de la face; la sélection quasi exclusive des génotypes $+/ b$ est possible dans le sexe mâle où très peu de reproducteurs sont retenus. Cet exemple d'épistasie mériterait des études complémentaires.

\section{INTRODUCTION}

Dans la race ovine Bleu du Maine, on a montré que la couleur noire est due à un seul facteur $b$, récessif par rapport à son allèle normal + , qui lui, ne modifie, pas la coloration blanche de la laine (LAUVERGNE, I96I). Cette étude prolongeait celles de Dry (1924, rg26), qui avait travaillé sur la race anglaise Wensleydale, race qui aurait certaines origines communes avec la race Bleu du Maine.

Dans ces 2 races les animaux noirs homozygotes $b / b$ sont impitoyablement écartés de la reproduction, ce qui devrait conduire à la disparition rapide de ce caractère, si, par ailleurs, les deux autres génotypes $+/+$ et $+/ \mathrm{b}$ ne subissaient pas de sélection préférentielle.

Or, pas plus Dry (r943) dans la race Wensleydale, que nous-mêmes dans la race Bleu du Maine, n'observons la disparition des noirs. Cela peut s'expliquer par le fait que les hétérozygotes $+/ b$ ont un avantage sélectif sur les homozygotes 
$+1+$; on constate en effet que la plupart, sinon la totalité des béliers, s'avèrent hétérozygotes.

Afin de préciser ce phénomène de sélection génotypique on a essayé, à partir des données du troupeau Chol, des populations permettant d'expliquer l'évolution de la fréquence des naissances noires.

\section{LES DONNÉES}

De I950, - date du début de la formation du troupeau -, à I960, on a trouvé 278 reproductrices que 1'on a pu classer en 3 générations de femelles $\left(F_{0}, F_{1}, F_{2}\right)$ comprenant respectivement $v_{0}, v_{1}, v_{2}$, individus; pour chacune de ces générations on connaissait le nombre de mises-bas $\left(\mathrm{N}_{1}, \mathrm{~N}_{2}, \mathrm{~N}_{3}\right)$ et la fréquence des naissances noires $\left(n_{1}, n_{2}, n_{3}\right)$, tableau $\mathrm{I}$.

TABLEAU I

Les données du troupeau ChOLLET-Molines

\begin{tabular}{|c|c|c|c|c|c|c|}
\hline \multicolumn{2}{|c|}{ Femelles } & \multicolumn{3}{|c|}{ Descendants } & \multirow{2}{*}{$\begin{array}{l}\text { Fréquence } \\
\text { des noirs }\end{array}$} & \multirow{2}{*}{$q^{\prime}$} \\
\hline Génération & Nombre & Blancs & Noirs & Total & & \\
\hline$F_{0}$ & $\nu_{0}=97$ & $6: 9$ & $n_{1}=15$ & $\Sigma_{1}=66^{\prime} t$ & 0,0678 & 0,1356 \\
\hline$F_{1}$ & $v_{0}=12 f$ & 559 & $n_{2}=6.7$ & $\mathrm{~N}_{2}=626$ & 0,1070 & 0,2140 \\
\hline $\mathrm{F}_{2}$ & $v_{2}=5 \overline{5}$ & 178 & $n_{3}=31$ & $\mathrm{~N}_{3}=209$ & 0,1483 & 0,2966 \\
\hline
\end{tabular}

Cette présentation fait apparaître des générations séparées de femelles, alors que les mâles, au nombre d'une dizaine seulement, ont été accouplés à des femelles de générations différentes; on verra plus loin comment on peut négliger cette imbrication.

On remarque une évolution très nette de la fréquence des noirs, qui passe de 7 p. Ioo à I $5 \mathrm{p}$. Ioo en 3 générations.

Le premier modèle d'étude est un modèle déterministe qui confond fréquence génique et probabilité ; c'est le plus simple ; il doit nous fournir une idée de la valeur de certains paramètres. Le test statistique ne pourra s'envisager qu'à partir d'un modèle stochastique qui, lui, tiendra compte du petit nombre de reproducteurs. dans chaque génération. C'est dans cet ordre que nous allons exposer notre étude : modèle déterministe, modèle stochastique, test statistique.

\section{LE MODÈLE DÉTERMINISTE}

\section{Io Les hypothèses}

La seule force susceptible de faire varier la fréquence génique est la sélection génotypique. On peut en effet négliger mutations, vu le petit nombre de générations, et migrations, en prenant certaines précautions.

(1) Voir : Remerciements. 
On est amené à supposer constants les coefficients de sélection que, pour une discussion, on peut notrer, $\sigma_{1}, \sigma_{2}, \sigma_{3}$, pour $+/+,+/ b$ et $b / b$ respectivement.

Ches les femelles tout d'abord on aura $\sigma_{3}=0$, puisque tous les sujets noirs sont éliminés; la sélection favorisant toujours les hétérozygotes on pourra noter $\sigma_{1}=k, \sigma_{2}=\mathrm{I}$ avec $0<k<\mathrm{I}$.

Chez les mâles, la sélection pratiquée n'a pas la même intensité que chez les femelles car le nombre de béliers employés est 30 fois inférieur au nombre de brebis.

DRY (I943) dans le troupeau d'UNDERLEY ne trouvait que des mâles hétérozygotes; c'est à une conclusion analogue que nous aboutissons dans le troupeau CHOLLLET-MOLINES, à une exception près, peut-être.

On simplifiera donc légitimement le modèle en supposant tous les mâles hétérozygotes $(+/ b)$; l'évolution de la fréquence génique se fera uniquement dans la population des reproductrices femelles. On voit que l'on a surmonté, de cette manière, la difficulté que présentait l'imbrication des générations due à l'emploi des mêmes béliers sur diverses générations femelles.

\section{$2^{\circ}$ La formule de récurrence}

On l'obtient en faisant d'abord le produit symbolique des fréquences gamètiques mâles et femelles en $\mathrm{F}_{n}$, puis en appliquant les coefficients de sélection génotypiques:

$$
(\mathrm{r} / 2 b+\mathrm{r} / 2+)\left(q_{n} b+p_{n}+\right) \quad \text { donne } \quad 0,5 p_{n}+/+; 0,5 \div / b ; 0,5 q_{n} b / b
$$

pour les fréquences génotypiques à la naissance. Ces génotypes vont constituer la génération $F_{n+1}$ après une sélection définie par les coefficients $k$ pour $+/+, \mathrm{I}$ pour $+/ b$ et o pour $b / b$.

Les fréquences génotypiques en $\mathrm{F}_{n+1}$ deviennent

$$
\frac{0,5 p_{n} k}{0,5+0,5 p_{n} k} \text { pour }+/+, \frac{0,5}{0,5+0,5 p_{n} k} \text { pour }+/ b \text { et o pour } b / b
$$

Dans ces conditions la fréquence génique $q_{n+1}$ de $b$ en $\mathrm{F}_{n+1}$ est la moitié de la fréquence génotypique de $+/ b$, soit :

$$
q_{n+1}=\frac{\mathrm{I}}{2} \frac{\mathrm{I}}{\mathrm{I}+k\left(\mathrm{I}-q_{n}\right)} .
$$

\section{$3^{\circ}$ L'étude de l'équilibre}

On peut facilement savoir si $q_{n}$ admet une limite en faisant $q_{n}=q_{n+1}$ dans l'équation (I). Ce qui nous ramène analytiquement à étudier, dans le plan des $q_{n}$, $q_{n+1}$ l'intersection d'une hyperbole d'équation (I) avec la première bissectrice. La seule solution acceptable sera celle qui est comprise entre o et $I$; on l'appellera $\hat{\imath}$ :

$$
\hat{q}=\frac{k+\mathrm{I}-\sqrt{\mathrm{I}+k^{2}}}{2 k}
$$


La disposition relative de la première bissectrice et de l'hyperbole fait que cet équilibre est un équilibre stable (fig. I).

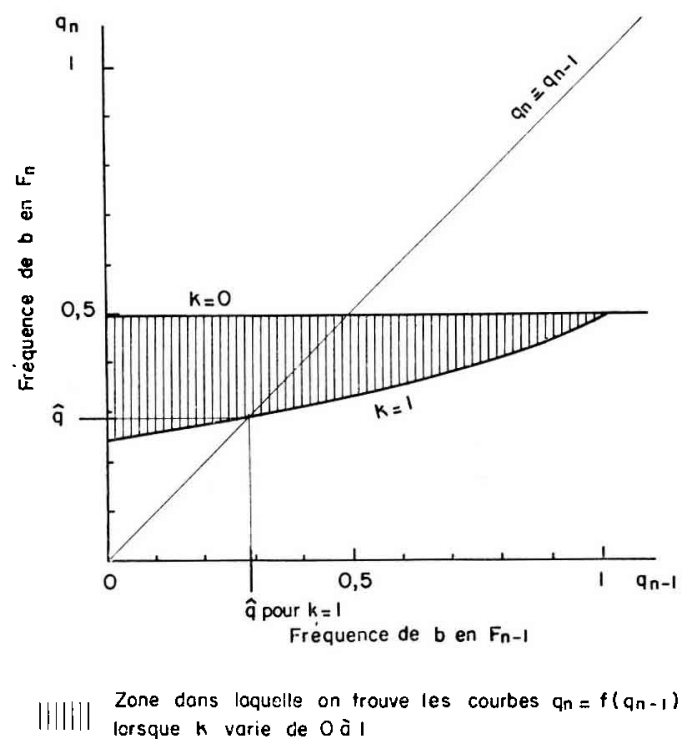

FIG. I - Représentalion graphique de la fréquence génique dans une génération en fonction de la fréquence dans la génération précédente

La valeur d'équilibre est fonction de $k$; elle pourra prendre toutes les valeurs comprises entre 0,5 (pour $k=0$ ) et 0,29 (pour $k=\mathrm{I}$ ) ; ces deux conditions correspondent, la première à l'élimination totale des $+/+$ chez les femelles, la seconde à l'absence de sélection préférant les hétérozygotes aux homozygotes.

\section{$4^{\circ}$ Le choix du parametre $\mathrm{k}$ :}

On remarque une coïncidence entre le chiffre $\hat{q}=0,29$ pour $k=\mathbf{I}$ et l'observation de $I 5 \mathrm{p}$. Ioo de noirs dans la descendance de $F_{2}$, qui correspondrait à une fréquence génique de 0,30 . Comme d'autre part dans le troupeau ChOL,ET-Mol,INES en formation on n'a pas pu exercer une forte sélection sur les femelles, il semble raisonnable d'adopter le chiffre de $k=\mathrm{I}$; on aura alors la formule de récurrence simplifiée

$$
q_{n+1}=\frac{I}{2} \cdot \frac{I}{2-q_{n}} .
$$

\section{IE MODĖLE STOCHASTIQUE}

Ce modèle va différer du modèle déterministe par un terme aléatoire dût au fait que les reproductrices d'une certaine génération sont en nombre limité $\nu_{n+1}$ et tirées au sort parmi les femelles à laine blanche ayant chacune la probabilité I/ $\left(2-q_{n}\right)$ d'être hétérozygote dans notre système de sélection (MALECOT, I959). 
La fréquence des hétérozygotes parmi les $v_{n+1}$ femelles sera une aléatoire de moyenne $\mathrm{I} /\left(2-q_{n}\right)$ et d'écart-type :

$$
\sqrt{\frac{\mathrm{I}-q_{n}\left(\mathrm{I}-\frac{\mathrm{I}}{2-q_{n}}\right)}{\nu_{n+1}} .}
$$

La fréquence génique de $b$ parmi les $v_{n+1}$ femelles n'est que la moitié de cette fréquence ; $q_{n+1}$ a donc pour moyenne $r / 2\left(2-q_{n}\right)$ et pour écart-type :

$$
\frac{I}{2} \sqrt{\frac{\frac{I}{2-q_{n}}\left(\mathrm{I}-\frac{\mathrm{I}}{2-q_{n}}\right)}{\nu_{n+1}}}=\sigma_{q_{n+1}} \text {. }
$$

Il apparaît done que $q_{n}$ et $q_{n+1}$ sont liés en chaîne de MARKofF :

$$
q_{n+1}=\frac{\mathrm{I}}{2} \frac{\mathrm{I}}{\left(2-q_{n}\right)}+\varepsilon_{n+1} \sigma_{q_{n+1}}
$$

où $\varepsilon_{n+1}$ est une aléatoire réduite $(O, I)$ qui suit une loi que l'on peut assimiler à une loi laplacienne.

La résolution de cette équation ne sera simple que si l'on admet l'hypothèse que l'arc d'hyperbole de la figure I peut être considéré comme une droite entre les points d'abscisse 0,13 et 0,29 . Cette droite doit passer par $M(\hat{q}, \hat{q})$; on obtiendra son équation en cherchant son coefficient angulaire. Un artifice de calcul souvent employé en mécanique peut consister à retrancher membre à membre les deux équations :

On obtient :

$$
q_{n+1}=\frac{I}{2\left(2-q_{n}\right)} \quad \text { et } \quad \hat{q}=\frac{I}{2(2-\hat{q})} .
$$

$$
q_{n+1}-\hat{q}=\frac{I}{2}\left(\frac{I}{2-q_{n}}-\frac{\mathrm{I}}{2-\hat{q}}\right)=\frac{\mathrm{I}}{2} \frac{q_{n}-\hat{q}}{\left(2-q_{n}\right)(2-\hat{q})} .
$$

On remplace alors le dénominateur par une valeur numérique en faisant une approximation pour $q_{n}$; l'erreur qui en découle n'est pas grande ; dans notre cas, on peut poser $q_{n}=0,2$ :

$$
\frac{\mathrm{I}}{2\left(2-q_{n}\right)(2-\hat{q})}=h=\frac{\mathrm{I}}{2 \times \mathrm{I}, 8 \times \mathrm{I}, 7} \# \mathrm{O}, \mathrm{I} 63
$$

La formule (3) peut alors s'écrire :

$$
q_{n+1}-\hat{q}=h\left(q_{n}-\hat{q}\right)+\varepsilon_{n+1} \sigma_{q_{n+1}} .
$$

C'est un processus de MARKoff linéarisé.

\section{TEST DES HYPOTHÈSES}

Le test des hypothèses suppose que l'on puisse comparer les $q$ observés aux $q$ obtenus par le calcul ; on doit alors tenir compte du fait que l'on ne connaît la fréquence de $b$ chez les femelles qu'à travers la descendance de ces femelles, c'est- 
à-dire qu'on va en avoir une estimée, entachée d'une certaine erreur aléatoire ; il s'agira ensuite de combiner ces diverses aléatoires pour aboutir à une équation fournissant les estimations de $q$ dans l'hypothèse de sélection que nous avons avancée, et de les comparer aux estimations des fréquences existantes.

\section{$I^{0}$ Estimation des fréquences existant chez les femelles}

Elle se fait à partir du nombre de sujets noirs observés dans les $\mathrm{N}_{n+1}$ descendants des $v_{n}$ femelles de $F_{n}$ croisées à des mâles qui sont tous hétérozygotes.

A chaque naissance peut être adjointe une aléatoire indicatrice du nombre des noirs :

$$
Z \begin{array}{ll}
0 ; & \mathrm{x}-\frac{q_{n}}{2} .
\end{array}
$$

Le nombre de noirs est une aléatoire $\mathrm{R}_{n+1}$ somme de $\mathrm{N}_{n+1}$ aléatoires $Z$, on a alors :

$$
\mathrm{E}\left(\mathrm{R}_{n+1}\right)=\mathrm{N}_{n+1} \mathrm{E}(Z)=\mathrm{N}_{n+1} \frac{q_{n}}{2}
$$

La variable aléatoire:

$$
q_{n}^{\prime}=\frac{2 \mathrm{R}_{n+1}}{\mathrm{~N}_{n+1}}
$$

qui a pour espérance mathématique $q_{n}$ peut être prise comme estimation de $q_{n}$; elle a pour variance :

$$
\sigma_{q^{\prime}}^{2}=\frac{q_{n}\left(2-q_{n}\right)}{\mathrm{N}_{n+1}}
$$

et suit approximativement une loi laplacienne ; on peut donc écrire :

$$
q_{n}^{\prime}-q_{n}=\alpha_{n} \sigma_{q_{n}^{\prime}}^{\prime},
$$

$\alpha_{n}$ étant une variable laplacienne réduite.

\section{$2^{\circ}$ Estimation des fréquences géniques du modèle stochastique :}

On a 5 aléatoires laplaciennes réduites $\alpha_{1}, \alpha_{2}, \alpha_{3}, \varepsilon_{1}, \varepsilon_{2}$ que l'on supposera indépendantes ; leur densité de probabilité conjointe est proportionnelle à

$$
e^{-1 / 2\left(\alpha_{i}^{2}+\alpha_{1}^{2}+\alpha_{2}^{2}+\varepsilon_{1}^{2}+\varepsilon_{2}^{2}\right)} \text {. }
$$

L'estimation des $q_{0}, q_{1}, q_{2}$ se fera en rendant maximum cette probabilité, c'est-à-dire en rendant minimum la somme des carrés des variables (équivalence de la méthode du maximum de vraisemblance avec la méthode des moindres carrés pour les lois de Laplace).

L'expression à minimiser s'écrit :

$$
\begin{aligned}
\lambda=\left[\frac{q_{1}-q-h\left(q_{0}-\hat{q}\right)}{\sigma_{q_{1}}}\right]^{2}+\left[\frac{q_{2}-q-h\left(q_{1}-\hat{q}\right)}{\sigma_{q_{2}}}\right]^{2} \\
+\quad+\left[\frac{q_{0}^{\prime}-q_{0}}{\sigma_{q_{0}^{\prime}}}\right]^{2}+\left\lfloor\frac{q_{1}^{\prime}-q_{1}}{\sigma_{q_{1}^{\prime}}}\right]^{2}+\left[\frac{q_{2}^{\prime}-q_{2}^{\prime}}{\sigma_{q_{2}^{\prime}}}\right]^{2} .
\end{aligned}
$$


En dérivant par rapport à $q_{0}, q_{1}$ puis $q_{2}$ et en remplaçant les $q^{\prime}$ par leurs estimées $\left(\frac{n_{1}}{\mathrm{~N}_{1}}, \frac{n_{2}}{\mathrm{~N}_{2}}, \frac{n_{3}}{\mathrm{~N}_{3}}\right)$ et les $\sigma_{q}$ et $\sigma_{i}$, par leurs valeurs numériques fonctions de ces estimées, on obtient le système :

$$
\left\{\begin{aligned}
33, \mathrm{I} q_{1}-267,4 q_{0}+27,5 & =0 \\
-\mathrm{I} 4,3 q_{2}+369,3 q_{1}-33, \mathrm{I} q_{0}-8 \mathrm{I}, 3 & =0 \\
\mathrm{I} 29 q_{2}-\mathrm{I} 4,3 q_{1} & -33,8=0
\end{aligned}\right.
$$

qui se résoud en donnant les estimées suivantes pour $q_{0}, q_{1}, q_{2}$, respectivement : $0, \mathrm{I} 33,0,243,0,289$.

\section{$3^{\circ}$ Comparaison des estimées obtenues par les 2 méthodes}

Dans le tableau 2 on a adopté comme intervalles de confiance plus ou moins deux fois l'écart-type. Pour une loi laplacienne cet intervalle contient $95 \mathrm{p}$. roo de la probabilité. On admet comme précédemment que nos lois binomiales ont un comportement quasi laplacien. La figure 2 sur laquelle on a porté les données du tableau 2 montre l'évolution des fréquences observées et calculées au cours des 3 générations étudiées. On a également représenté les intervalles de confiance.

TABLEAU 2

Estimées des fréquences existantes, comparées aux estimées obtenues par la méthode du maximum de waisemblance dans le modele stochastique

\begin{tabular}{|c|c|}
\hline $\begin{array}{c}\text { Estimées } \\
\text { des fréquences existantes }\end{array}$ & $\begin{array}{l}\text { Estimées des fréquences } \\
\text { du modèle stochastique }\end{array}$ \\
\hline $\begin{array}{l}q_{0}^{\prime}=0,136 \pm 0,039 \\
q_{1}^{\prime}=0,214 \pm 0,049 \\
q_{2}^{\prime}=0,297-\ldots, 098\end{array}$ & $\begin{array}{l}q_{0}=0,133 \\
\left.q_{1}=0,2+3+0,0\right)_{1}^{\prime} \\
q_{2}=0,289 \perp 0,0337\end{array}$ \\
\hline
\end{tabular}
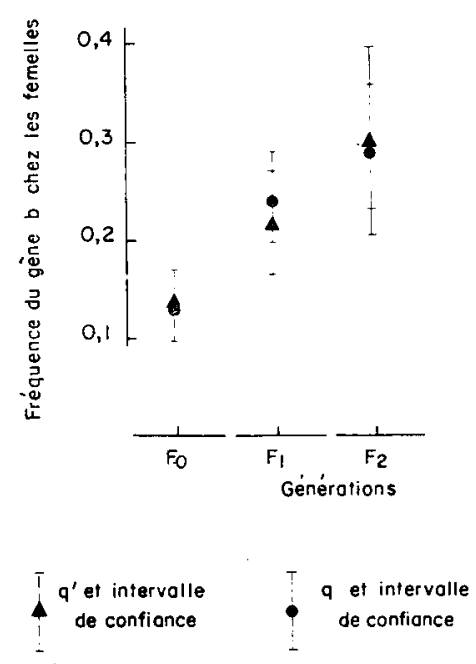

I' IG. 2 - Estimées des fréquences géniques observées, comparées aux estimées données par le maximum de vraisemblance (q) dans trois générations de femelles 


\section{DISCUSSION}

Les intervalles de confiance des estimées se recouvrent très largement (fig. 2); on peut donc très raisonnablement accepter l'hypothèse proposée : tous les mâles sont $+b ; k=\mathrm{x}$ chez les femelles.

Il faut toutefois souligner que nous avons un nombre très restreint de générations. Ce qui fait que des hypothèses un peu différentes pourraient tout aussi valablement êtres testées et acceptées (fréquence moins élevée de $b$ chez les mâles, accompagnée de sélection plus intense chez les femelles).

Néanmoins cet ajustement des données observées aux données calculées nous confirme l'importance du phénomène de sélection génotypique exercé par les éleveurs à leur insu. A UNDERLEY, DRY (I924) observait même 20,3 p. Ioo de naissances noires ce qui correspondrait à un avantage sélectif, accordé aux femelles hétérozygotes $+/ b$ par rapport aux homozygotes $+1+$.

\section{CONCLUSION}

Ainsi se confirme 1'hypothèse d'une interaction génétique : le facteur $b$, à l'état hétérozygote, intensifie la couleur bleue qui est sous l'influence d'un autre système génétique inconnu.

Pour que les éleveurs ne continuent pas une sélection aussi décevante, il conviendrait de n'employer que des béliers homozygotes $+/+$, testés sur leur descendance.

L'explication physiologique d'une interaction entre plusieurs systèmes génétiques reste à trouver.

Rę̧u en mai 1962.

\section{REMERCIEMENTS}

Mr Chollet-Molines, éleveur à Soudon par Cheffes-sur-Sarthe (Maine-et-Loire), s'est donné la peine d'extraire de son livre d'élevage les données sans lesquelles nous n'aurions pu entreprendre cette étude. Qu'il trouve ici l'expression de notre gratitude.

L'auteur tient particulièrement à remercier le Pr G. MALECOT de la Faculté des Sciences de Lyon pour l'aide qu'il lui a apportée dans l'élaboration du modèle stochastique et du test statistique.

\section{SUMMARY}

THE EVOLUTION OF A GENE FREQUENCY IN “ BLEU DU MAINE 》 SHEEP

"Bleu du Maine " sheep are white fleeced with blue-black heads and feet. They are similar in certain respects to the English Wensleydale breed with wich they share a common ancestry.

Certain animals are entirely black, a colour governed by a genetical factor which is recessive to the one for white. All black $b / b$ sheep are prevented from reproducing, but this form of selection is not sufficient to cause these genotypes to disappear. 
According to DRY (1926, 1943) we have been led to expect that the heterozygotes $+/ b$ offer a selective advantage over the $+1+$. In the flock studied this selection seemed to be clear and strong, particularly with the males. With regard to the females, if certain hypotheses are accepted, $q_{n+1}$ the frequency of gene $b$ in $F_{n+1}$ generation is given by the equation :

$$
q_{n+1}=\mathrm{I} / 2 \frac{\mathrm{I}}{2-q_{n}} .
$$

However, since these data are not numerous, the statistical test may be applied only by making use of a stochastic model. In this way one arrives at a MARKOFF linear process given by the formula:

$$
q_{n+1}=\hat{q}+h\left(q_{n}-\hat{q}\right)+\varepsilon_{n+1} \sigma q_{n+1} \text {. }
$$

The statistical test is applied to the evolution of genetical frequency over three successive generations of females and is used by applying the rule of maximum of likelihood, once the frequency existing in the three generations has been estimated.

The hypothesis submitted that all the males are $+/ b$ individuals and that there is no selection among the females has not been invalidated. Equilibrium frequency is quite reached by the 3 rd generation and is manifested by a rate of 15 p. 100 of black sheep at birth.

\section{RÉFÉRENCES BIBLIOGRAPHIQUES}

Dry F. W., I924. The genetics of the Wensleydale breed of seheep. I. The occurence of blacks lambs, an examination of flock records. J. Gen., 14, 203-20S.

DRY F. W., 1926. Colour inheritance in the Wensleydale breed of shep. J. Text. Inst., 17, I180-1 I86

Dry F. W., I943. The genetics of the Wensleytale breed of sheep. III. Arithmetical aspects of selection. J. Gen., 45, $265 \cdot 268$.

Lauvergne J. J., rg6r. Sur le déterminisme génétique de la couleur noire dans la race "Bleu du Maine ». Sem. Hop. Paris (Ann. Gen.), 2, 47-52.

Malecot G., r959. Les modèles stochastiques en génétique de population. Pub. Inst. Sta. Paris, 8, 173210. 